Pathologe 2008 · [Suppl 2] 29:240-244

DOI 10.1007/s00292-008-1078-z

Online publiziert: 10. Oktober 2008

(c) Springer Medizin Verlag 2008

\author{
K. Hauptmann ${ }^{1} \cdot$ I. Melcher ${ }^{2} \cdot$ K.-D. Schaser ${ }^{2}$ \\ ${ }^{1}$ Institut für Pathologie, Charité-Universitätsmedizin Berlin \\ ${ }^{2}$ Centrum für Muskuloskelettale Tumoren der Klinik für \\ Unfallchirurgie, Charité-Universitätsmedizin Berlin
}

\title{
Differenzialdiagnose intramedullärer Osteosarkome
}

Osteosarkome bilden eine morphologisch und biologisch äußerst heterogene Tumorgruppe. Ohne genaue Kenntnis des radiologischen Aspekts ist die richtige Einordnung der unterschiedlichen histologischen Bilder einer Knochenbiopsie kaum möglich. In vielen Fällen sind die vorliegenden radiologischen Veränderungen bereits wegweisend, wenn nicht manchmal sogar eindeutig. So ist das konventionelle Röntgenbild von hochmalignen Osteosarkomen so charakteristisch, dass in mehr als der Hälfte der Fälle keine andere Differenzialdiagnose infrage kommt. Deshalb stellen hochmaligne Osteosarkome in der Regel kein großes diagnostisches Problem dar. Dennoch kann die Einordnung des histologischen Bildes wegen des großen Variantenreichtums dieser Tumorgruppe in manchen Fällen schwierig sein. Dagegen werden niedrigmaligne Osteosarkome häufig fehlinterpretiert. Die zentralen „Low-grade-Osteosarkome“ sind we- niger aggressiv und haben ein deutlich geringeres Metastasierungspotenzial. Mit einem Anteil von weniger als $5 \%$ an allen Osteosarkomen sind sie so selten, dass die Erfahrungen mit dieser Tumorgruppe nur gering sind [8]. Die Interpretation der radiologischen Befunde und der histologischen Veränderungen ist problematisch und gelingt manchmal erst nach Kenntnis des weiteren klinischen Verlaufs.

\section{Definition und Klassifizierung}

Definitionsgemäß handelt es sich bei einem Osteosarkom um einen Tumor, dessen Tumorzellen Osteoid oder Knochen bilden. In vielen hochmalignen Osteosarkomen ist die osteoblastische Komponente jedoch nur diskret nachweisbar. Stattdessen stehen andere Differenzierungen stark im Vordergrund. So imponiert in den Biopsien häufig eine chondroide Matrix oder auch eine fibröse,

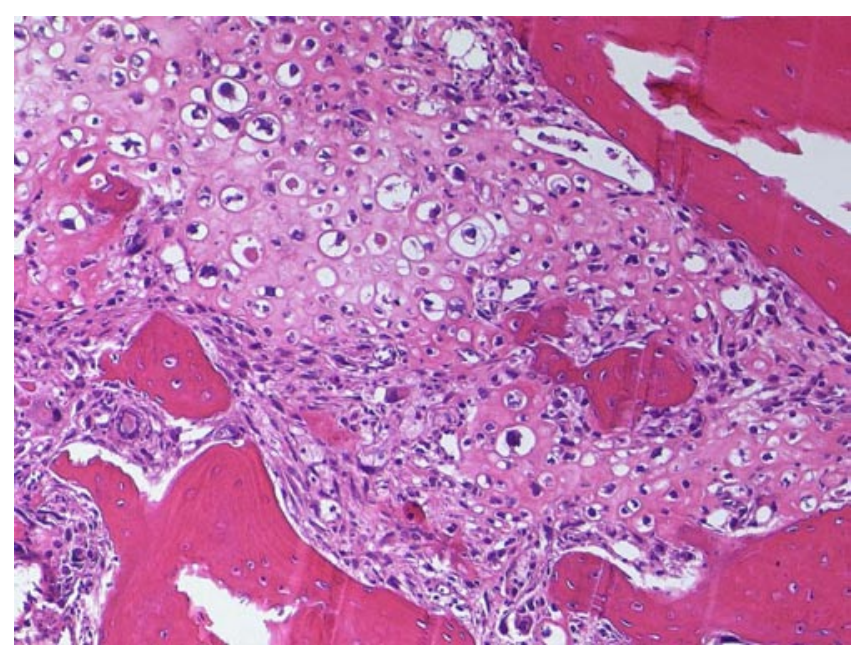

Abb. $1<$ Hochmalignes Osteosarkom, chondroblastischer Subtyp mit Resten ortsständiger Spongiosa und erheblichen Dyskaryosen der atypischen Knorpelzellen (HE-Färbung, Vergr. 300:1) teilweise auch spindelzellige Komponente, wie man sie in Weichteilsarkomen findet. Diese Matrix kann im Tumor sogar dominieren. Nach der überwiegend gebildeten Matrix (über 50\% in der Probebiopsie) in einem Tumor wird der Subtyp (osteoblastisch, chondroblastisch, fibroblastisch) definiert. Dieser hat bisher jedoch biologisch keinerlei Bedeutung und spielt auch in der Planung des therapeutischen Vorgehens keine Rolle $[1,5,7]$. Das histologische Bild, das aus diesen variablen Differenzierungen resultiert, ist in einem "High-grade-Osteosarkom“ aus diesem Grund oft vielgestaltig, erinnert manchmal aber auch an einen Frakturkallus.

Auch die Gruppe der „Low-gradecentral-Osteosarkome" ist sehr variantenreich. Der einzelne Tumor zeigt jedoch meist ein gleichförmiges Muster mit großer Ähnlichkeit zu einem benignen Knochentumor, wodurch die hohe Rate an Fehlinterpretationen zu erklären ist $[2,3,8]$.

\section{Zentrale hochmaligne Osteosarkome}

Das konventionelle hochmaligne Osteosarkom ist der häufigste maligne Knochentumor. Es manifestiert sich meist in der 2. Lebensdekade, wobei mehr Jungen als Mädchen betroffen sind. Das Auftreten vor dem 10. Lebensjahr und nach Abschluss des Wachstums ist vermehrt mit anderen Knochenanomalien assoziiert [11]. Betroffen sind in der Mehrzahl der Fälle die Knochen mit den größten Wachstumsraten, und statistisch gesehen sind Kinder 


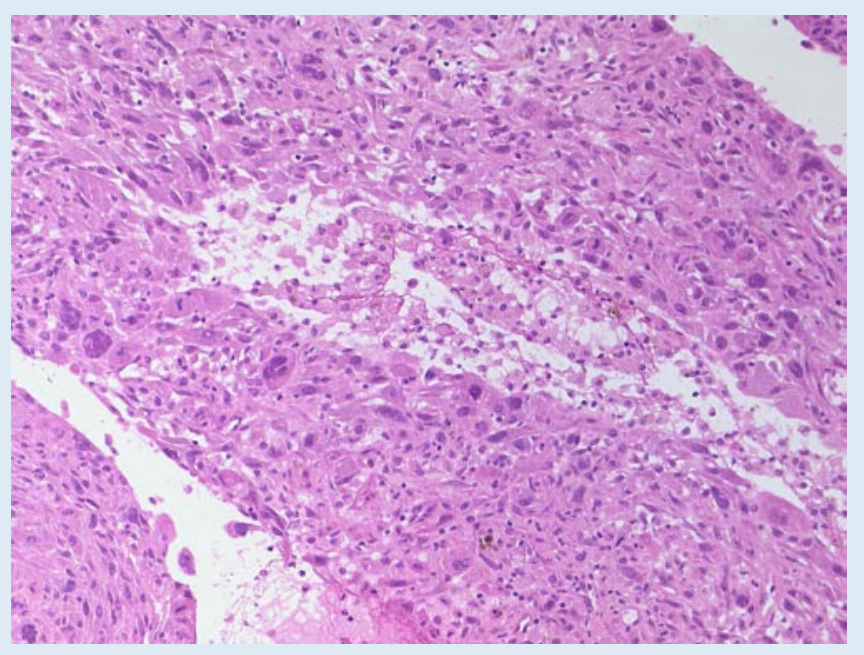

Abb. $2 \Delta$ Zellreiche zystenwandartige Strukturen mit einer Auskleidung durch atypische Zellen in einem teleangiektatischen Osteosarkom. Deutliche Dyskaryosen und atypische Mitosen (HE-Färbung, Vergr. 250:1)

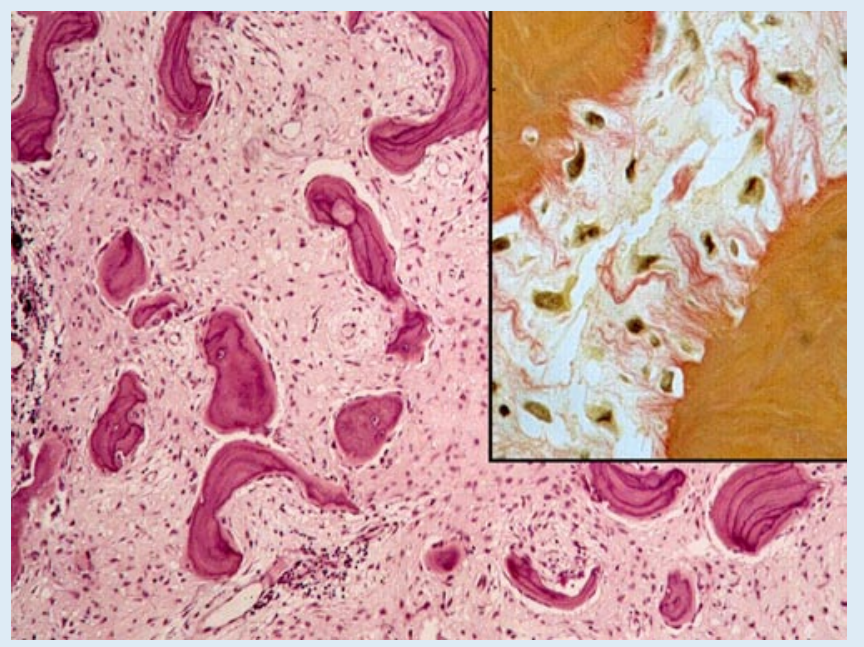

Abb. 4 \ Niedrigmalignes Osteosarkom ähnlich einer fibrösen Dysplasie. Zellreiches fibröses Stroma, typische Spikulae (Insert) und irreguläre Knochenbälkchen (HE-Färbung, Vergr. 200:1)

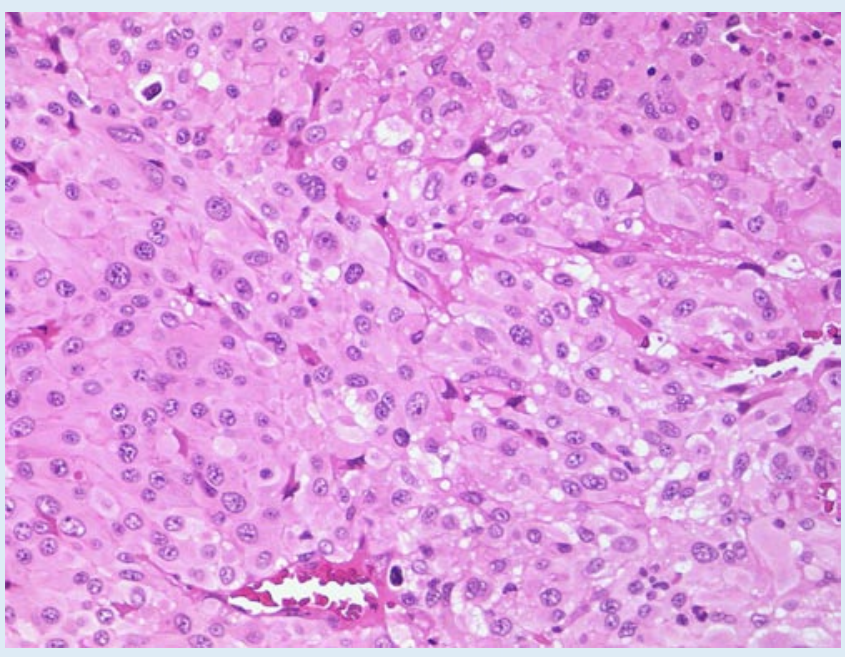

Abb. $3 \Delta$ Epitheloides Osteosarkom mit soliden Zellformationen und diskreten Osteoidabscheidungen. Große polygonale Zellen mit breitem hellem Zytoplasmasaum und vesikulären Kernen (HE-Färbung, Vergr. 400:1)

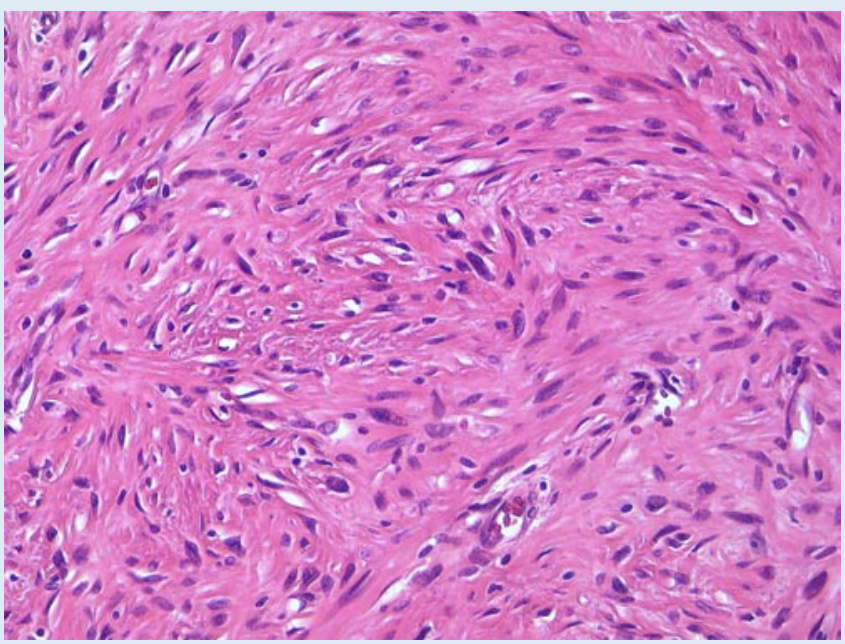

Abb. $5 \Delta$ Leichte zelluläre Atypien der spindeligen Zellen in einem niedrigmalignen Osteosarkom, einem desmoplastischen Fibrom ähnlich (HE-Färbung, Vergr. 400:1) oder Jugendliche mit einem Osteosarkom durchschnittlich größer - eine Beobachtung, die auch im Tierreich gilt [4].

Unter dem Begriff des zentralen hochmalignen Osteosarkoms werden das konventionelle Osteosarkom mit seinen unterschiedlichen Subtypen sowie mehrere Varianten wie das teleangiektatische, das kleinzellige, das epitheloide und das riesenzellreiche Osteosarkom zusammengefasst.

\section{Konventionelles Osteosarkom}

Das konventionelle Osteosarkom weist radiologisch meist metaphysäre, unscharf begrenzte Osteolysen und herdförmige Sklerosierungen auf. Die meisten Tumoren haben zum Zeitpunkt der Diagnose die Kortikalis durchbrochen und besitzen einen extraossären Tumoranteil [5].

\section{Osteoblastisches Osteosarkom}

Mikroskopisch ist das klassische osteoblastische Osteosarkom meist zellreich. Die Tumorzellen sind teils polygonal seltener spindelförmig. Sie besitzen pleomorphe, hyperchromatische Zellkerne. Mitosen, darunter atypische Kernteilungsfiguren, kommen regelmäßig vor. Die Tumorzellen bilden gitterförmig Os- teoid oder flächenhaft unreifen mineralisierten Knochen.

\section{Chondroblastisches Osteosarkom}

Chondroblastische Osteosarkome zeichnen sich dagegen durch atypische Knorpelformationen aus. Diese können so dominieren, dass Verwechslungen mit einem Chondrosarkom möglich wären, wenn man den klinischen Aspekt (radiologischer Befund, Alter des Patienten) unbeachtet lässt. Typische Osteoidabscheidungen sind manchmal nur ganz diskret nachweisbar. Die Tumorchondrozyten zeigen Kerngrößenschwankungen und prominente Nukleolen. Als Zeichen eines ag- 
Pathologe 2008 · [Suppl 2] 29:240-244

DOI 10.1007/s00292-008-1078-z

(c) Springer Medizin Verlag 2008

\section{K. Hauptmann · I. Melcher · K.-D. Schaser Differenzialdiagnose intramedullärer Osteosarkome}

\section{Zusammenfassung}

Intramedulläre hochmaligne Osteosarkome sind aggressive Tumoren mit hohem Metastasierungspotenzial. Die zahlreichen Subtypen und Varianten dieser Tumoren führen oft zu differenzialdiagnostischen Problemen, weswegen eine kritische Zusammenschau der morphologischen und radiologischen Befunde erforderlich ist. Niedrigmaligne zentrale Osteosarkome unterscheiden sich von den hochmalignen Osteosarkomen grundlegend. Sie haben große Ähnlichkeiten zu benignen Knochentumoren, sodass sie schwer zu diagnostizieren sind. Um Rezidive zu vermeiden, ist eine onkologische suffiziente, meist weite Resektion erforderlich. Die Metastasierungsneigung dieser Tumoren ist viel geringer als die der hochmalignen Osteosarkome.

\section{Schlüsselwörter}

Intramedulläres Osteosarkom · Hochmaligne . Niedrigmaligne

\section{Differential diagnosis of intramedullary osteosarcomas}

\begin{abstract}
Intramedullary high-grade osteosarcomas are aggressive tumors with a high metastatic capacity. The many subtypes and variants of these tumors often make the differential diagnosis difficult. Therefore, a critical review of both radiology and histomorphology is mandatory. Low-grade central osteosarcomas are a completely different tumor group. Due to their strong similarity to benign bone tumors they are frequently misdiagnosed as such. The correct diagnosis followed by radical surgery, however, is essential to reduce the high risk of local recurrences, typically seen for of these lesions. Unlike conventional high grade osteosarcoms, distant metastases are much less frequent than in low grade osteosarcomas.
\end{abstract}

Keywords Intramedullary osteosarcoma · High-grade . Low-grade gressiven, infiltrierenden Wachstums werden ortsständige Spongiosabälkchen nach Art eines „chondrosarcoma permeation pattern“ umschlossen (• Abb. 1).

\section{Fibroblastisches Osteosarkom}

Dagegen stehen bei einem fibroblastischen Subtyp kollagenfaserhaltige Abschnitte wie bei einem Fibrosarkom im Vordergrund.

\section{Sklerosierendes Osteosarkom}

Besonders hervorzuheben sind die Osteosarkome mit starker Sklerosekomponente, da sie in kleinen Biopsien meist schwierig zu diagnostizieren sind. Gelegentlich kann eine bimssteinartige Struktur radiologisch auf einen solchen Tumor hinweisen. Histologisch sind die Biopsien auffällig zellarm, und es überwiegen Areale mit flächenhaften knöchernen Grundsubstanzabscheidungen. Besonders tückisch ist, dass die wenigen eingeschlossenen Tumorzellen normalen Osteozyten zum Verwechseln ähnlich sind, ein Befund, der bereits von Jaffe als „normalization phenomenon" beschrieben wurde [6].

\section{Teleangiektatisches Osteosarkom}

DasteleangiektatischeOsteosarkom ist eine Variante des hochmalignen Osteosarkoms, dessen Häufigkeit mit 2-12\% angegeben wird [16]. Es lässt sich sowohl radiologisch als auch histologisch von den konventionellen Subtypen abgrenzen, ähnelt aber benignen Läsionen, wie der aneurysmatischen Knochenzyste, dem Riesenzelltumor bzw. einem Hämangioendotheliom $[12,16]$. Auch das mit 16 Jahren angegebene durchschnittliche Erkrankungsalter ist für die Differenzialdiagnostik wenig hilfreich. Gleiches gilt für den radiologischen Befund, der oft nicht von dem einer aneurysmatischen Knochenzyste zu unterscheiden ist. In vielen Fällen imponiert eine asymmetrische, stark lytische, zystische Struktur. Der Knochen ist erheblich aufgetrieben, und der Prozess kann expansiv über die Kortikalis hinaus das angrenzende Weichgewebe infiltrieren, ohne dass ein Neokortex gebildet wird.

Auch das mikroskopische Bild mit Nachweis von blutgefüllten, zystenwandartigen Formationen erinnert auf den ers- ten Blick an eine aneurysmatische Knochenzyste oder einen Gefäßtumor. Die Zystenwandungen sind jedoch sehr zellreich, wobei 2 Zellkomponenten vorkommen: Neben reichlich typischen osteoklastären Riesenzellen zeigt die zweite mononukleäre Komponente deutliche zelluläre und nukleäre Atypien und auch Mitosen (• Abb. 2).

Ein weiteres wichtiges diagnostisches Kriterium ergibt sich aus der Beurteilung der die Hohlraumbildungen auskleidenden Zellen. Im Gegensatz zur aneurysmatischen Knochenzyste, für die eine flache endothelähnliche Zellschicht charakteristisch ist, fällt auch hier eine atypische, sarkomatöse Zelllage auf. Außerdem ist die Proliferationsfraktion (MIB-1) ausgesprochen hoch. Die Osteoidbildung ist in diesen Tumoren meist äußerst spärlich, kann auch gänzlich fehlen.

Die Prognose der teleangiektatischen Osteosarkome wurde in der Vergangenheit unterschiedlich bewertet. Entgegen früheren Untersuchungen, die einen ungünstigeren Verlauf beschrieben, geht man heute von einer besseren Prognose als bei konventionellen Osteosarkomen aus. Man vermutet, dass dies auf ein besseres Ansprechen (Response) auf Polychemotherapie (wahrscheinlich bedingt durch die hohe Wachstumsfraktion bei insgesamt geringer Tumorzellzahl) dieser Tumoren zurückzuführen ist $[1,7,16]$. Als prognostisch ungünstiger Faktor ist jedoch der höhere Prozentsatz an pathologischen Frakturen anzusehen, der durch die ausgeprägten Osteolysen bedingt ist.

\section{Kleinzelliges Osteosarkom}

Kleinzellige Osteosarkome stellen nur einen Anteil von etwa 1-2\% aller Osteosarkome dar und sind differenzialdiagnostisch besonders schwierig zu beurteilen. Deswegen ist in diesem Fall eine sehr intensive Diskussion der klinischen, radiologischen und morphologischen Befunde erforderlich. Aufgrund der geringen mineralisierten Matrixbildungen weist bei diesen Tumoren das radiologische Bild nicht unbedingt auf ein Osteosarkom hin. Die Differenzialdiagnose schließt sowohl eine Osteomyelitis als auch ein Ewing-Sarkom ein. Von Interesse für den Pathologen kann auch der intraoperative, makrosko- 
pische Aspekt eines mit Knochentumoren erfahrenen Operateurs sein. So sprechen ein sehr großer extraossärer Anteil und eine weiche, bröcklige Konsistenz des Tumors eher für das Vorliegen eines EwingSarkoms.

Histologisch sieht man einen kleinzelligen Tumor mit hyperchromatischen Zellkernen und ungewöhnlichen knöchernen Grundsubstanzabscheidungen $[9,13]$. Leider ist die Immunhistochemie für die differenzialdiagnostische Abgrenzung gegenüber einem Ewing-Sarkom wenig richtungsweisend, da auch für kleinzellige Osteosarkome eine membranäre positive Reaktion für $\mathrm{CD} 99$ beschrieben wird. Der Nachweis von spindelzelligen Abschnitten sollte unbedingt Beachtung finden, da diese wesentlich auf ein kleinzelliges Osteosarkom hinweisen. In diesen Fällen sollte immer mittels RT-PCR oder FISH nach der Translokation $t(11 ; 22)$ der Ewing-Gruppe gesucht werden, da selbst dann, wenn der Tumor mineralisiert ist, aber die spezifische $t(11 ; 22)$-Translokation aufweist, von einem Ewing-Sarkom ausgegangen und entsprechend therapiert werden muss $[5,7]$.

\section{Epitheloides Osteosarkom}

Ähnlich problematisch gestaltet sich die Diagnostik einer weiteren, ebenfalls seltenen Variante hochmaligner Osteosarkome, dem epitheloiden Osteosarkom. In diesen Tumoren steht die zelluläre Komponente stark im Vordergrund, wohingegen nach einer Matrixproduktion sorgfältig gesucht werden muss. Meist imponieren große polygonale dicht gelagerte solide Zellen, die große Ähnlichkeit zu epithelialen Tumoren haben ( $\bullet$ Abb. 3), da sogar adenoide Formationen ausgebildet werden können. Die Zellkerne sind stark vergrößert und können prominente $\mathrm{Nu}$ kleolen haben.

Vorraussetzung für die Diagnose eines epitheloiden Osteosarkoms ist ein kongruenter Befund im konventionellen Röntgenbild. Bei älteren Patienten muss unbedingt die Metastase eines gering differenzierten Karzinoms ausgeschlossen werden. Die Immunhistologie ist nicht ausreichend, da ein Teil der epitheloiden Osteosarkome sogar eine aberrante Zytokeratinexpression aufweist [15].
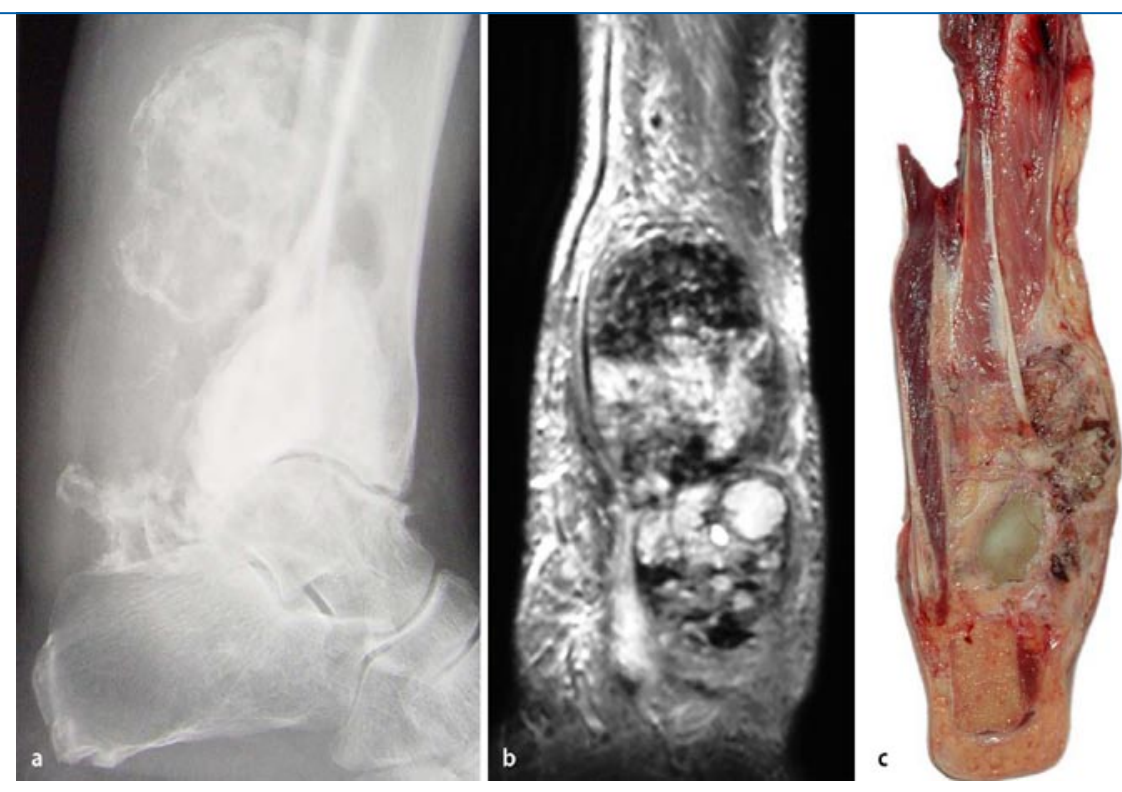

Abb. $6 \Delta$ Rezidiv eines niedrigmalignen Chondroblastoma-like-Osteosarkom der distalen Tibia mit ausgedehntem Weichteilanteil. Zustand nach Zementauffüllung des Primärtumors. Zentrale Osteolysen und Sklerosierungen. Deutliche Randsklerose im konventionellen Röntgenbild (a) und in der MRT (b). c Tumorgesamtebene nach Unterschenkelamputation

\section{Riesenzellreiches Osteosarkom}

Die riesenzellreiche Variante des Osteosarkoms zeichnet sich durch einen hohen Anteil osteoklastärer Riesenzellen aus, sodass Beziehungen zum Riesenzelltumor, zum teleangiektatischen Osteosarkom und auch zur aneurysmatischen Knochenzyste bestehen. Die zweite zelluläre Komponente bilden mononukleäre Zellen mit deutlichen Zell- und Kernatypien und eindeutige gitterförmige Osteoid abscheidungen. Nach den eigenen Erfahrungen enthält die Probebiopsie meist zusätzliche Abschnitte mit einer weiteren Differenzierung wie z. B. eine chondroblastische Komponente. Das Alter des Patienten und das radiologische Bild passen besser zu einem konventionellen Osteosarkom als zu einem Riesenzelltumor, der kaum vor dem 16. Lebensjahr beobachtet wird und sich durch eine exzentrische, epiphysär-metaphysäre Osteolyse auszeichnet. Ist der tumortragende Knochen kein Röhrenknochen und der Patient älter, kann die differenzialdiagnostische Abgrenzung zu einem malignen Riesenzelltumor problematisch sein.

\section{Zentrale niedrigmaligne Osteosarkome}

Nach wie vor sind die Kriterien für die Diagnostik zentraler niedrigmaligner Osteosarkome unscharf und nicht gut definiert. Radiologisch können sowohl stark osteolytische Läsionen als auch dichte Sklerosierungen imponieren. Häufig deuten eine unscharfe Begrenzung, eine unterbrochene Periostreaktion oder eine umschriebene Destruktion der Kompakta auf einen lokal aggressiveren Tumor hin.

Histologisch liegen meist fibroossäre Läsionen vor $[2,8]$. Die Tumorzellen bilden in unterschiedlicher Menge unreife, irregulär konfigurierte mineralisierte Knochenbälkchen, die von einem hochdifferenzierten fibrösen Stroma umgeben werden, sodass das Bild mit einer fibrösen Dysplasie zu verwechseln ist (• Abb. 4). Oder aber es steht eine fibröse, spindelzellige Komponente im Vordergrund, ähnlich wie bei einem desmoplastischen Fibrom (• Abb. 5). Andererseits wurden auch Tumoren mit einer chondroiden Matrix ähnlich einem Chondroblastom (- Abb. 6 a-c) oder mit einer ausgeprägten osteoblastischen Komponente ähnlich einem Osteoblastom beschrieben $[8,14]$. Die Vielfalt der morphologischen Muster führte deshalb zu einer Unterteilung in zahlreiche Subtypen. 


\section{Hauptreferate: Orthopädische Pathologie}

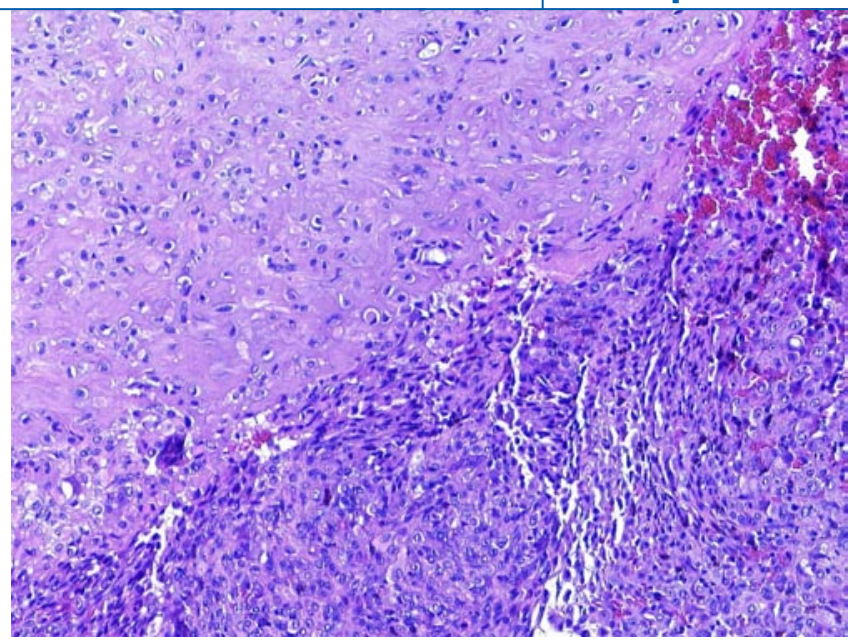

Abb. $7<$ Die Histologie des Rezidivs des Patienten aus Abb. 6 mit reichlich Chondroblasten in ausgedehnten chondroiden Grundsubstanzbildungen in der linken oberen Bildhälfte. Übergang in zellreichere Abschnitte mit mehr länglichen Zellen (rechts unten; HE-Färbung, Vergr. 200:1)

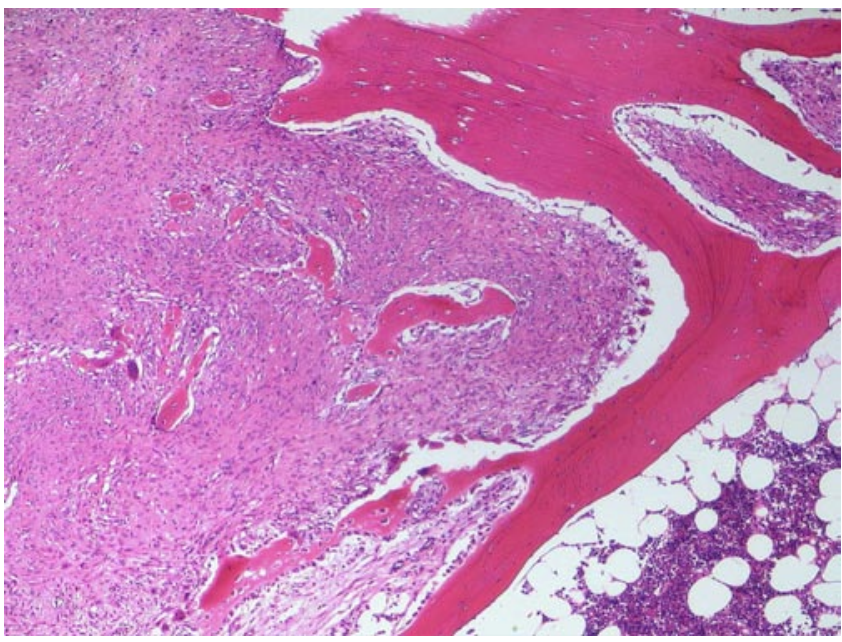

Abb. $8<$ Infiltrierendes Wachstum eines niedrigmalignen Osteosarkoms "desmoplastic fibroma-like" mit Umwachsen von präexistenten Knochenbälkchen (HE-Färbung, Vergr. 200:1)

Im Unterschied zu ihren „look alikes“ sind ein stärkerer Zellreichtum, der Nachweis von zellulären Atypien sowie Mitosen die entscheidenden Diagnosekriterien für ein zentrales Low-grade-Osteosarkom. Es können jedoch auch viel diskretere Veränderungen, beispielsweise die Art der Grundsubstanzbildung, auf das Vorliegen eines sarkomatösen Tumors hinweisen. So sollten auch ungewöhnlich ausgedehnte knöcherne Matrixbildungen, der so genannte „Lace-like-Osteoid“, in einem vermeintlichen Osteoblastom mit dem radiologischen Befund abgeglichen werden.

Flächenhafte chondroide Grundsubstanzbildungen können einen Hinweis auf ein „Chondroblastoma-like-Osteosarkom" sein, insbesondere dann, wenn der typische epitheloide Charakter der Chondroblasten fokal verloren gegangen ist und stattdessen spindelzellige Areale nachweisbar sind (• Abb. 7).

Wichtig für die Diagnose ist auch die Beurteilung der Umgebung des Tumors.
So kann als Hinweis auf ein infiltrierendes Wachstum der ortständige spongiöse Knochen von der Läsion umschlossen werden (- Abb. 8).

Eine Infiltration oder die fokale Destruktion der Kompakta ist immer suspekt auf einen aggressiven Tumor. Deshalb ist eine sorgfältige Musterung der HaversKanäle für die endgültige Diagnose unterstützend. Der durchschnittliche Altersgipfel eines Low-grade-Osteosarkoms liegt in der 3. Lebensdekade. Damit ist das Manifestationsalter etwas höher als bei Patienten mit einem hochmalignen konventionellen Osteosarkom. Die Verteilung der Tumoren innerhalb des Skelettsystems entspricht der von hochmalignen Tumoren, wobei Männer und Frauen gleichhäufig betroffen sind. Die Prognose der niedrigmalignen Tumoren ist günstiger. Voraussetzung für einen exzellenten klinischen Verlauf ist eine radikal-chirurgische Therapie, da eine intraläsionale Kürettage oder auch marginale Resektion des Tumors zwangsläu- fig das Auftreten von Rezidiven nach sich zieht. Die Rezidive treten im Durchschnitt 3 Jahre postoperativ auf, und ein Drittel davon rezidiviert als High-grade-Osteosarkom, oder es entstehen fokale Dedifferenzierungen wie bei einem parossalen Osteosarkom. Bei 25\% der Patienten treten später pulmonale oder knöcherne Metastasen auf $[2,3,8,10]$.

\section{Korrespondenzadresse PD Dr. K. Hauptmann \\ Institut für Pathologie, \\ Charité-Universitätsmedizin Berlin \\ Charitéplatz 1, 10117 Berlin \\ kathrin.hauptmann@charite.de}

Interessenkonflikt. Die korrespondierende Autorin gibt an, dass kein Interessenkonflikt besteht.

\section{Literatur}

1. Bacci G, Bertoni F, Longhi A et al. (2003) Neoadjuvant chemotherapy for high-grade central osteosarcoma of the extremity. Histologic response to preoperative chemotherapy correlates with histologic subtypes of the tumor. Cancer 97: 3068-3075

2. Bertoni F, Bacchini P, Fabbri N et al. (1993) Osteosarcoma. Low-grade intraosseous-type osteosarcoma histologically resembling parosteal osteosarcoma, fibrous dysplasia and desmoplastic fibroma. Cancer 71: 338345

3. Choong PF, Pritchard DJ, Rock MG et al. (1996) Low grade central osteogenic sarcoma: a long-term follow up of 20 patients. Clin Orthop Relat Res 322: 198-206

4. Cotterill SJ, Wright CM, Pearce MS et al. (2004) Stature of young people with malignant bone tumors. Pediatr Blood Cancer 42: 59-63

5. Dorfmann HD, Czerniak B (1998) Bone tumors. Mosby, St Louis/MO

6. Jaffe HL (1960) Intracortical osteogenic sarcoma. Bull Hosp Jt Dis 21: 189-197

7. Klein MJ, Siegal GP (2006) Osteosarcoma: Anatomic and histologic variants. Am J Clin Pathol 125: 555-581

8. Kurt AM, Unni KK, McLeod RA, Pritchard DJ (1990) Low grade intraosseous osteosarcoma. Cancer 65: 14181428

9. Nakajama H, Sim FH, Bond JR et al. (1997) Small cell osteosarcoma of bone: review of 72 cases. Cancer 79: 2095-2106

10. Ogose A, Hotta T, Emura I et al. (2000) Repeated dedifferentiation of low-grade intraosseous osteosarcoma. Hum Pathol 31:615-618

11. Porter DE, Holden ST, Steel CM et al. (1992) A significant proportion of patients with osteosarcoma may belong to Li-Fraumeni cancer families. J Bone Joint Surg Br 74: 883-886

12. Saito T, Oda Y, Kawaguchi K et al. (2005) Five-year evolution of a telangiectatic osteosarcoma initially managed as an aneurysmal bone cyst. Skelet Radiol 34 290-294

13. Sim FH, Unni KK, Beabout JW et al. (1979) Osteosarcoma with small cells simulating ewing's tumor. J Bone Joint Surg Am 61: 207-215

14. Tani T, Okada K, Shoji K et al. (2000) Osteoblastomalike osteosarcoma. Skelet Radiol 29: 656-659

15. Unni KK (ed) (1998) Osteosarcoma of bone. In: Bone tumors. Churchill Livingstone, New York/NY

16. Weiss A, Khoury JD, Hoffer FA et al. (2007) Telangiectatic osteosarcoma: The St. Jude children's research hospital's experience. Cancer 109: 1627-1637 